\title{
Surprisingly fast cooling in graphene-based van der Waals stacks
}

\author{
Hailin Peng ${ }^{{ }^{*}}$ and Zhenjun Tan ${ }^{1,2}$
}

A thorough understanding of nanoscale heat flow in heterostructures is crucial for future multifunctional nanodevices based on 2D materials. For example, the thermal exchanges based on hot-carrier cooling pathways and time in graphene devices have been rigorously investigated, and these studies provide guidance for the development of electronic and optoelectronic applications $[1,2]$. Furthermore, defect-assisted acoustic phonon scattering [3], intrinsic optical phonon (OP) cooling [4], and interlayer Coulomb coupling [5] are the three primary relaxation mechanisms in diffusive, suspended, and decoupled multilayer graphene, respectively. However, perfect supported or encapsulated graphene, in which electrons are coupled to remote polar phonon modes, is different from the other types of graphene mentioned above, and it lacks sufficient experimental research. As a uniaxial dielectric, hexagonal boron nitride (hBN) sustains a large number of hyperbolic phonon-polaritons (HPPs). Thus, the hBN/graphene heterostructure provides a perfect research platform for nanoscale heat transfer of van der Waals heterostructures. Recently, Koppens et al. [6] and Placais et al. [7] independently verified highly surprisingly fast heat flow within van der Waals heterojunctions through different solid experiments, which made great progress in the study of nanoscale thermal transport.

Koppens et al. [6] identified the picosecond relaxation times and dynamics of the hyperbolic cooling process in graphene heterostructures. In order to measure the ultrafast carrier dynamics of hBN-encapsulated graphene (illustrated in Fig. 1a), they performed pump-probe photocurrent measurements on the hBN-encapsulated, Hall-bar-shaped, exfoliated graphene devices. To exclude the disorder assisted cooling, the long mean-free path and high carrier mobility of graphene were extracted firstly. Based on the energy transfer rate and the pump-probe experimental data, the cooling dynamics with a cooling timescale of $\tau_{\text {calc }}=C_{n} \frac{T_{\mathrm{e}}-T_{\mathrm{L}}}{Q}$ were obtained, where $C_{n}$ is the electronic heat capacity of graphene and $Q$ is the energy transfer rate.

It was found that the hyperbolic cooling model of hBN semi-quantitatively reproduces the experimentally observed trends for the entire range of investigated carrier densities and lattice temperatures. As illustrated in Fig. $1 b$, the cooling slows down for lower lattice temperatures and around the Dirac point, which is mainly attributed to the smaller energy transfer rate $Q$. It should be noted that the cooling was slower when being encapsulated with very thin hBN flakes, because of the lower density of hyperbolic modes in thinner hBN.

Noise thermometry, combined with Joule heating, is another powerful tool to investigate energy relaxation. At the same time, Placais et al. [7] gave strong evidence for this new and ultra-efficient cooling pathway by using sensitive noise thermometry based on bilayer graphene transistors, demonstrating the emission of HPPs by outof-equilibrium electron-hole pairs. A crucial difference between the low and high bias cooling mechanisms lies in their opposite dependences on carrier concentration, as shown in Fig. 1c. This indicates two dominating cooling processes: intraband electron-electron interactions at low field and interband HPP emission at high field. The sudden jump in emissivity (Fig. 1d) cannot be understood within a thermal scheme because emissivity at fixed doping shows a smooth dependence on temperature. This significant increase in emissivity indicates an ultra-efficient, strongly out-of-equilibrium process.

In these intriguing $2 \mathrm{D} \mathrm{hBN}$ /graphene heterostructures, efficient coupling between graphene carriers and HPPs in hBN layer opens up thermal pathways, through which heat is efficiently drained away from the device. The

${ }^{1}$ Center for Nanochemistry, College of Chemistry and Molecular Engineering, Peking University, Beijing 100871, China

${ }^{2}$ Academy for Advanced Interdisciplinary Studies, Peking University, Beijing 100871, China

*Email: hlpeng@pku.edu.cn 

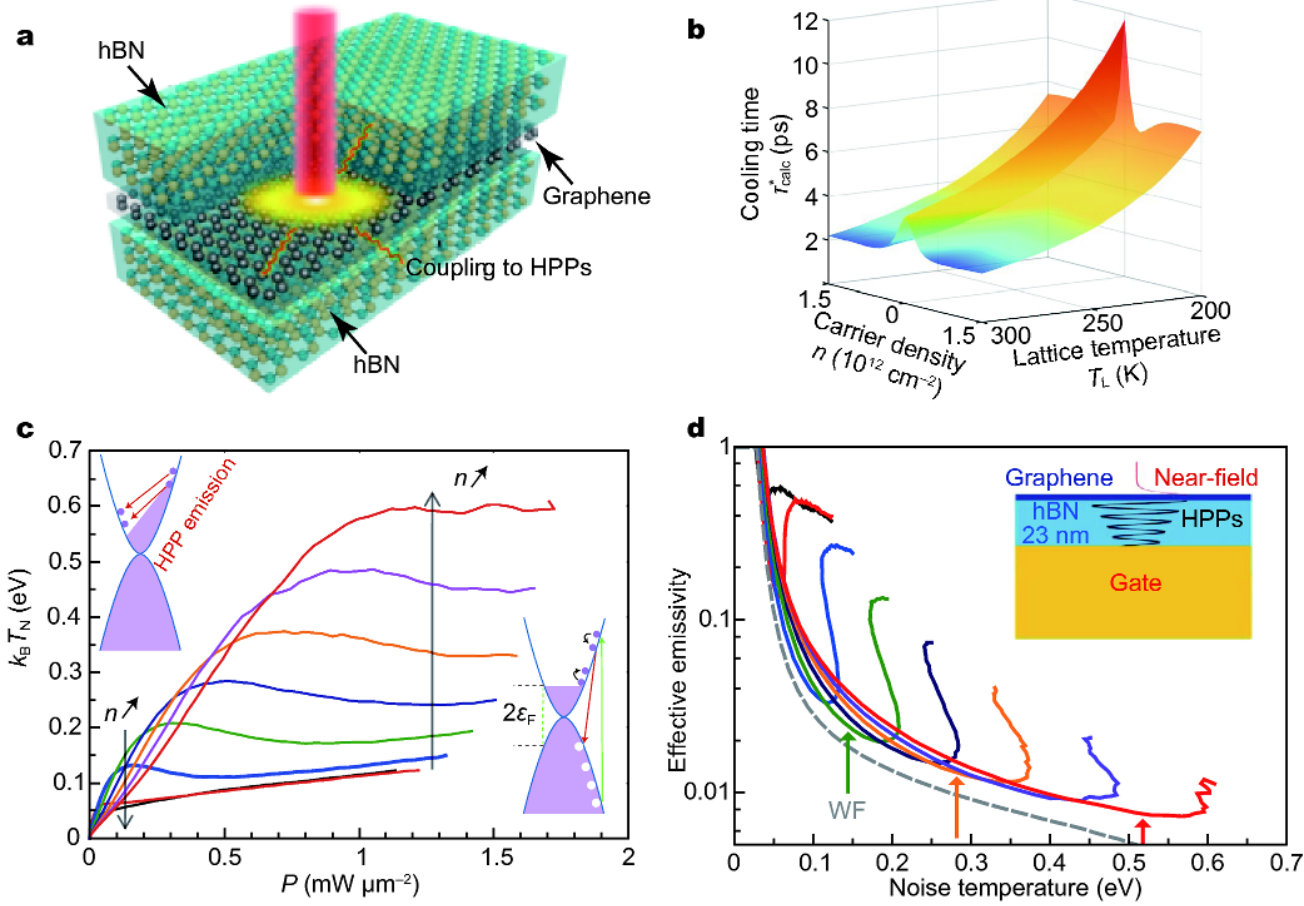

Figure 1 (a) Schematic representation of out-of-plane heat transfer in hBN-encapsulated graphene. (b) Both experimentally and theoretically, the cooling time was found to vary with the carrier density and lattice temperature. (c) Noise temperature as a function of Joule heating. Arrows indicate the doping dependence of the noise temperature, which is positive in a low field and negative in a high field. (d) HPP emissivity analysis of the noise temperature-Joule power data shown in (c). Inset: sketch of the radiative heat transfer by HPPs. These pictures are from references [6,7]. Copyright 2017, Nature publishing group.

magnitude of this HPP relaxation time is in the order of picoseconds. These studies on nanoscale thermal transport in 2D heterostructures hold significant implications on thermal managements, optoelectronics, thermoelectrics and data communications.

Received 30 December 2017; accepted 2 January 2018; published online 12 January 2018

1 Song JCW, Rudner MS, Marcus CM, et al. Hot carrier transport and photocurrent response in graphene. Nano Lett, 2011, 11: 4688-4692

2 Koppens FHL, Mueller T, Avouris P, et al. Photodetectors based on graphene, other two-dimensional materials and hybrid systems.
Nat Nanotechnol, 2014, 9: 780-793

3 Song JCW, Reizer MY, Levitov LS. Disorder-assisted electronphonon scattering and cooling pathways in graphene. Phys Rev Lett, 2012, 109: 106602

4 Kampfrath T, Perfetti L, Schapper F, et al. Strongly coupled optical phonons in the ultrafast dynamics of the electronic energy and current relaxation in graphite. Phys Rev Lett, 2005, 95: 187403

5 Mihnev MT, Tolsma JR, Divin CJ, et al. Electronic cooling via interlayer Coulomb coupling in multilayer epitaxial graphene. Nat Commun, 2015, 6: 8105

6 Tielrooij KJ, Hesp NCH, Principi A, et al. Out-of-plane heat transfer in van der Waals stacks through electron-hyperbolic phonon coupling. Nat Nanotechnol, 2017, 13: 41-46

7 Yang W, Berthou S, Lu X, et al. A graphene Zener-Klein transistor cooled by a hyperbolic substrate. Nat Nanotechnol, 2017, 13: 47-52 